\title{
Interfacial energy issues in ceramic particulate reinforced metal matrix composites
}

\author{
BRIJ K DHINDAW \\ Metallurgical and Materials Engineering, Indian Institute of Technology, Kharagpur 721 302, India
}

\begin{abstract}
One major scientific issue that needs to be resolved and understood in order to design ceramic particle reinforced metal matrix composites is the interfacial energy state between the matrix and the reinforcement. Solid-solid interfacial energy between the particle and the matrix effects the final interface characteristics and also significantly influences the particle redistribution due to its effect on particle pushing engulfment by the melt interface. The paper analyses the physics behind the particle pushing and engulfiment by the solidifying interface considering models utilizing interfacial force as energy difference between the particle in the solid and particle in the liquid melt. Various methods of evaluating solid-solid interfacial energy have been discussed. Velocity of melt interface movement at which the particles are engulfed by the matrix referred to as critical velocity of the system under given conditions has been shown to be directly related to the interfacial energy. Critical appraisal of experiments to determine the critical velocity have been presented for aluminium matrix dispersed with zirconia particles. Advantages of carrying out experiments under $\mu \mathrm{g}$ environment have been pointed out.
\end{abstract}

Keywords. Composite; interface; energy; critical velocity; micro gravity.

\section{Introduction}

\subsection{Interface energy in composites}

Composite materials represent a new generation of materials with great potentials for novel properties, and can be tailored to specific engineering applications (Nair et al 1985; Oh et al 1987). Particle reinforced metal matrix composites can be produced by two major routes: melt processing and powder metallurgy. Compared with powder metallurgy melt processing has some important advantages, e.g. better matrix-particle bonding, easier control of matrix structure, simplicity and low cost of production. Mechanical properties of particle reinforced metal matrix composites (PRMMC) are not only a function of the nature of matrix and of the particle but also of the distribution of the particles with respect to the grains. An ideal solution will be one in which all particles are distributed intragranular and there are no particle agglomerates. One major scientific issue that needs to be understood and resolved in order to design composite components and their optimum processing conditions is the interface energy between matrix material and reinforcement (Rohatgi et al 1993). This needs to be understood when the matrix is in liquid and solid states. This understanding is necessary when the processing is through liquid melting route or powder processing rout. The interface energy is important when the particles are transferred from air to liquid melt (Kacar et al 1991) and from liquid to solid phase during the matrix solidification process (Omenyi and Newmann 1976). In both these cases a lowering of overall energy of the system is ideal for the stability of the composite. However, in most instances such possibility is hard to achieve and one has to look at the kinetics of the transfer process to evaluate the possibilities. However, solid-solid interface energy between the particle and the solidified matrix is an important parameter that needs to be accurately known to characterize the properties of the composite. In the present paper the specific issue of role of interfacial energy between the particle and the matrix in particle redistribution during liquid to solid transformation has been discussed in detail.

\subsection{Particle-melt interaction}

A particular situation during composite fabrication occurs when the solidifying interface meets the reinforcement particle. The particle can get redistributed in three ways depending upon whether the particle is pushed, engulfed or entrapped by the interface as shown in figure 1 (Juratzko et al 1998). The results are also the functions of morphology of interface. The interaction shown in figure 1 has been to some extent modelled and understood for the planar interfaces.

\section{Theoretical modelling}

\subsection{Critical velocity}

Over the past two decades, several theoretical models (UhIman et al 1964; Bolling and Cisse 1971; Chernov 
et al 1976; Stefanescu et al 1988, 1990, 1998) have been developed to predict the behaviour of an insoluble particle interacting with an advanced solid/liquid interface. Even though the experimental and theoretical research was lately focused on possible applications to metal matrix composites produced by casting (Stefanescu et al 1988; Kennedy and Clyne 1991) or spray forming techniques (Wu et al 1994; Lawrynowicz et al 1997), other applications of this concept have emerged over the years. One of the most recent applications of particle pushing/ engulfment is in the production of $\mathrm{Y}_{1} \mathrm{Ba}_{2} \mathrm{Cu}_{3} \mathrm{O}_{7-\delta}$ (123) superconductors (Endo et al 1996). One processing route is by growing 123 crystals from an under cooled liquid. The oxide melt contains $\mathrm{Y}_{2} \mathrm{Ba}_{1} \mathrm{Cu}_{3} \mathrm{O}_{5}$ (211) precipitates which act as flux pinning sites. These sites enhance the critical current density of the superconductor. Upon solidification the 211 precipitates segregate at the crystal growth front. Optimal properties can only be obtained if an uniform precipitate distribution is achieved. Another application of this concept is in the field of cryobiology (Korber et al 1985). The goal is the preservation of biological cells by maintaining a uniform concentration of salts during the freezing process of these cells.

The last non-metal related application to be listed is in the field of frost heaving of soils (Jackson and Chalmers 1958). The resulting expansion cracks the surface of the soil (frost heaving), the classic concern being with paved roads. The treatment of the problem can be reduced to the behaviour of soil particles (i.e. clay) in a water/ice matrix.

Nevertheless, the majority of research work involving particle pushing is aimed at metal matrix composites (MMCs). Here as already mentioned the driving force is to obtain a homogenous particle distribution. Consequently, the cooling conditions of cast MMCs must be

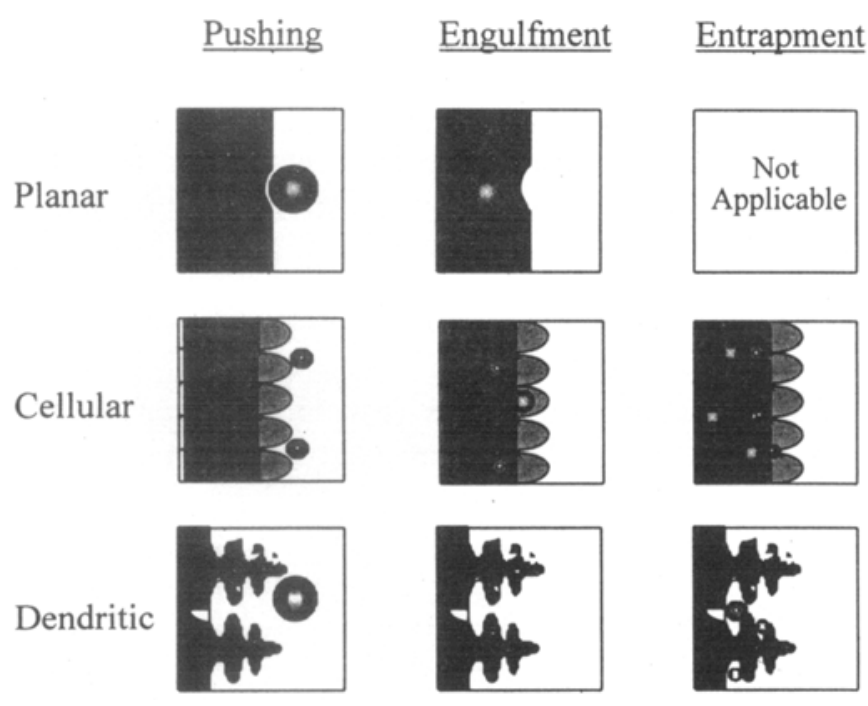

Figure 1. Particle-interface interaction. controlled so that the solidification velocity is higher than the critical velocity at which particles are engulfed by the solidifying interface. Only with a homogenous distribution of the reinforcement phase the improved mechanical properties of these materials can be fully exploited. The concept of 'critical velocity' has been discussed in several previous publications (Uhlman et al 1964; Bolling and Cisse 1971; Chernov et al 1976; Stefanescu et al 1988, 1990, 1998). To understand how interfacial energy between matrix and particle holds key to the calculation of critical velocity and vice versa, some current accepted analytical models derived for critical velocity evaluation are given in the following paragraph.

\subsection{Analysis of current models}

The analytical models approach this problem as a balance between the attractive viscous drag force and the repulsive interfacial force emanating from interfacial energies. There are two analytical models that use directly interfacial force between the solid particle and the matrix in the calculation of the critical velocity:

I. Potschke and Rogge Model (partly numerical) (Potschke and Rogge 1989):

$$
V=\frac{1 \cdot 3 \Delta \sigma_{0}}{\mu}\left[16\left(\frac{R}{a_{0}}\right)^{2} K^{\circ}\left(15 K^{\circ}+x\right)+x^{2}\right]^{-1 / 2}
$$

$V$ is the critical velocity for engulfment, $\Delta \sigma_{0}$ the difference between the interfacial energies of the liquid, solid matrix and particle, $\mu$ the liquid viscosity, $R$ the radius of the particle, $a_{0}$ the interatomic distance, $K^{\circ}$ the $K_{\mathrm{p}} / K_{\mathrm{L}}$, ratio of thermal conductivities of particle and liquid, respectively and $x$ a function of impurity concentration for pure metals, i.e. $x=0$.

II. Stefanescu et al (1998) Model. This model originated from the concept in Stefanescu et al (1998) model:

$$
V_{\mathrm{cr}}=\left(\frac{\Delta \gamma_{0} a_{0}^{2}}{3 \eta K^{*} R}\right)^{1 / 2}
$$

$V_{\text {cr }}$ is the critical velocity for engulfment, $\Delta \sigma_{0}$ the difference between the interfacial energies of the liquid, solid matrix and particle, $\mu$ the liquid viscosity, $R$ the radius of the particle, $a_{0}$ the interatomic distance and $K^{*}$ the $K_{\mathrm{p}} / K_{\mathrm{L}}$, ratio of thermal conductivities of particle and liquid, respectively.

There are other models based on Hamaker constant related to the Van der Waal type of forces between the solid particles and the interface of the solidifying melt. 
However, debilitating assumptions make calculations through those models approximate, without any prospects of further accuracy, because of measurement difficulties of the scientific issues involved. Further interfacial energy calculations cannot be verified with the help of above models.

\section{Interfacial energy calculations}

Most of the thermophysical parameters utilized in the models with repulsive force being surface energy are fairly accurately available. However, $\Delta \sigma_{0}$ values are difficult to calculate. In the above equations, $\Delta \sigma_{0}$ has been evaluated as the difference in the interfacial energies between particle-solid (Stefanescu et al 1998), $\sigma_{\mathrm{ps}}$ and the particle-liquid, $\sigma_{\mathrm{pl}}$. The force between two solids has been attributed to as $\Delta \sigma_{0}=\Delta \sigma_{\mathrm{ps}}-\Delta \sigma_{\mathrm{pl}}$ or $\Delta \sigma_{0}=\Delta \sigma_{\mathrm{ps}}-\Delta \sigma_{\mathrm{pl}}-\Delta \sigma_{\mathrm{sl}}$, where $\Delta \sigma_{0}=$ interfacial force. $\Delta \sigma_{\mathrm{ps}}=$ particle/solid interfacial energy, $\Delta \sigma_{\mathrm{pl}}=$ particle/ liquid interfacial energy.

An arbitrary value of $1 \mathrm{~N} / \mathrm{m}$ has been assumed (Stefanescu et al 1990) most of the times for $\Delta \sigma_{0}$. Since this assumption was based on general considerations and not on exact measurements it was not possible to accurately validate the models predicting the behaviour of an insoluble particle interacting with a solid-liquid interface. $\Delta \sigma_{0}$ could not be accurately characterized as there are no well established experimental techniques to measure the contact angle between the solids which is very important in finding the value of $\Delta \sigma_{p s}$ which influences the value of $\Delta \sigma_{0}$. In the following discussion the methods of calculating the interfacial energy are presented in detail. For calculating $\Delta \sigma_{\mathrm{ps}} ; \gamma_{\mathrm{PS}}$ and $\gamma_{\mathrm{PL}}$ are needed.

$\gamma_{\mathrm{PL}}$ can be calculated from Young's equation (Murr 1975):

$$
\gamma_{\mathrm{PL}}=\gamma_{\mathrm{PV}}-\gamma_{\mathrm{LV}} \cos \theta
$$

Both $\gamma_{\mathrm{PV}}$ and $\gamma_{\mathrm{LV}}$ are typically available in the literature, being directly measured for many systems. The contact angle $\theta$ can be measured using sessile drop experiments.

The evaluation of $\gamma_{\mathrm{PS}}$ is more complicated. A number of researchers have used the generic equation of state proposed by Omenyi et al (1981) for a two-component, three-phase system:

$$
\gamma_{12}=\frac{\left(\gamma_{13}^{1 / 2}-\gamma_{23}^{1 / 2}\right)^{2}}{1-K_{0}\left(\gamma_{13}^{1 / 2}-\gamma_{23}^{1 / 2}\right)}
$$

where 1, 2, 3 are three different phases in contact with each other, and $K_{0}$ is a material constant. For this problem the phases of interest are particle $(\mathrm{P})$, liquid $(\mathrm{L})$, solid (S), and vapour (V) phases; they can be used in any order. However, values of $K_{0}$ in above equation is quite often arbitrarily extrapolated from the experimental measurements of few parameters. Therefore, while this equation with some success has been used for organic systems it fails for metallic matrices.

Another approach for the calculation of $\gamma_{\mathrm{PS}}$ is by using the work of adhesion. The underlying assumption behind this approach is that when two solids that are in contact are separated, because of the creation of two new solid-vapour interfaces, the interface energy in the system is increased by the work of adhesion:

$$
\gamma_{\mathrm{PS}}=\gamma_{\mathrm{PV}}+\gamma_{\mathrm{SV}}-W_{\mathrm{ad}-\mathrm{PS}}
$$

In turn, the work of adhesion can be calculated from the Girifalco-Good relationship (Adamson 1982);

$$
W_{\mathrm{ad}-\mathrm{PS}}=2 \Phi \sqrt{\gamma_{\mathrm{PV}} \cdot \gamma_{\mathrm{SV}}}
$$

The coefficient $\Phi$ can have a value of $0 \cdot 24$. Then, if $\gamma_{\mathrm{PV}}$ and $\gamma_{\mathrm{SV}}$ are known, $\gamma_{\mathrm{PS}}$ can be calculated. Thus, an additional term must be added to the equations describing $\gamma_{\mathrm{PS}}$ :

$$
\gamma_{\mathrm{PS}}=\gamma_{\mathrm{PV}}+\gamma_{\mathrm{SV}}-W_{\mathrm{ad}}
$$

This analysis is valid assuming perfect contact between the matrix and the particle. Such contact is rather improbable for non-wetting systems. To account for the imperfect contact, this equation must be modified as follows:

$$
\gamma_{\mathrm{PS}}=\gamma_{\mathrm{PV}}+\gamma_{\mathrm{SV}}-a W_{\mathrm{ad}}
$$

where $a=0$ for no contact, and $a=1$ for perfect contact. It is reasonable to assume that for rough particles that are not wetted by the liquid, as is the case for most ceramic particles in contact with liquid metals, $a$ will be closer to zero than to one. The assumption $a=0$ is equivalent to $\boldsymbol{\Phi}=0$.

In many instances, $\gamma_{\mathrm{pv}}$ and $\gamma_{\mathrm{sv}}$ can be found in the literature. $\gamma_{S V}$ can also be calculated from the heat of sublimation (Murr 1975), with the equation:

$$
\gamma_{\mathrm{sv}}=0 \cdot 16 \Delta H_{\text {vap }}
$$

\section{Experimental techniques for interfacial energy $\gamma_{\mathrm{PS}}$ measurement}

\subsection{The sputtered film coating technique}

This approach (Murr 1975) is essentially an extension of equilibrating a liquid metal droplet on a ceramic substrate and measuring the contact angle between the two phases. Here the matrix substance can be sputter 
coated on the particle ceramic substrate, annealed and the contact angle of the resultant globule of the matrix on ceramic substrate measured with the help of scanning electron microscopy. This gives the interfacial energy in the same manner as for liquid drops on solid substrates in sessile drop technique.

\subsection{Powder metallurgy technique}

The powders of metal and ceramic are thoroughly mixed and vacuum sintered at temperatures higher than the melting point of the metallic phase (Kingrey 1954). Subsequently the resultant sintered mass would be annealed at lower temperatures for a long period of time. It is expected that at the grain boundaries between the ceramic grain and the metal a dihedral angle would be formed. However, both the above methods have not been seriously pursued so far as there are large number of difficulties yet to be overcome in terms of technique and calculations.

\section{Interfacial energy and critical velocity}

Considering critical velocity equation and assuming a particular matrix and particles of particular diameter the critical velocity is a direct function of the interfacial energy term as $\eta, K_{\mathrm{p}} / K_{\mathrm{l}} a_{0}$ become constants. However, in the experimental measurement under identical conditions different critical velocities have been found by different workers. Sometimes the values differ by an order of magnitude. The two major variables which can cause this difference are interfacial energy and interface shape being wrongly evaluated. The difference in the interfacial energy term can come from the surface characteristics of the particles used (although they can have the same chemical composition and preparation methodology) and because of interface characteristics between the particle and the matrix after solidification. There

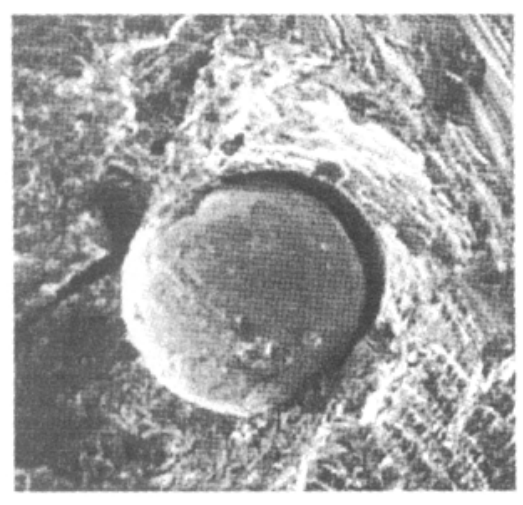

Figure 2. Scanning micrograph of fractured surface of Alzirconia composite, showing gap around the zirconia particle. may be for example gap between particle and matrix due to the presence of gases or impurities in the melt or due to altered wettability of the particle by the matrix. Figure 2 shows a typical case where the gap between the particle and the matrix is visible after solidification.

Therefore if the critical velocity is known accurately for a particular matrix and particles, the interface conditions can be characterized by calculation of the interfacial energy from the equation relating critical velocity with the variables. It can be understood that if the interface between the particle and the matrix is mostly as shown in figure 2 , then the resultant composite would have inferior mechanical properties. In such cases the interfacial energy between the particle and the matrix would be lower than that expected theoretically.

\section{Experimental measurement of critical velocity}

There are several conditions which need to be satisfied to get accurate experimental measurement of the critical velocity. These include inert particle-matrix system, spherical particles, convection free environment etc. For ground based experiments it is not possible to satisfy all of the criteria. For the ground base experiments aluminium (99.999\%) and zinc (99.95\%) were chosen (Juratzko et al 1998) as matrix materials because of the interest in these materials for MMCs.

Zirconia particles were selected because they reportedly ( $\mathrm{Li}$ 1994) do not react with aluminum up to temperatures of $900^{\circ} \mathrm{C}$ (table 1). Additionally $\mathrm{ZrO}_{2}$ is thermodynamically more stable than $\mathrm{Al}_{2} \mathrm{O}_{3}$. Thus no reducing reaction is expected below $900^{\circ} \mathrm{C}$. Finally, spherical zirconia particles were available, while it was not possible to find spherical $\mathrm{SiC}$ particles.

The experimental critical velocities for PET are given in table 2. The table also gives the values of critical velocity as obtained from the micro gravity environment

Table 1. Variation of contact angle with temperature for aluminium on two substrates (Li 1994).

\begin{tabular}{lcc}
\hline & \multicolumn{2}{c}{ Contact } \\
\cline { 2 - 3 } Substrate & $T=700^{\circ} \mathrm{C}$ & $T=1100^{\circ} \mathrm{C}$ \\
\hline $\mathrm{ZrO}_{2}$ & 150 & 87 \\
$\mathrm{SiC}^{2}$ & 160 & 58 \\
\hline
\end{tabular}

Table 2. Experimental critical velocities $(\mu \mathrm{m} / \mathrm{sec})$.

\begin{tabular}{|c|c|c|}
\hline \multirow[b]{2}{*}{ System } & \multicolumn{2}{|c|}{ Critical velocity $(\mu \mathrm{m} / \mathrm{sec})$} \\
\hline & Ground & $\mu \mathrm{g}$ \\
\hline $\begin{array}{l}\text { Al-zirconia }(500 \mu \mathrm{m} \text { dia }) \\
\text { Zn-zirconia }\left(500 \mu \mathrm{m}^{\prime} \text { dia }\right)\end{array}$ & $\begin{array}{l}1.9-2.4 \\
1.9-2.9\end{array}$ & $\begin{array}{c}0.5-1.0 \\
\text { not available }\end{array}$ \\
\hline
\end{tabular}


experiments conducted abroad space shuttle (Stefanescu et al 1998).

\section{Discussion}

The paper has presented results of critical velocity measurement from the best possible controlled experimental conditions. This has been achieved by design of the experimental set up and the choice of the matrix and the particles. It can be seen that even with such controlled conditions critical velocities at best could be determined as a range, however narrow the range may be. As argued earlier if all the physical parameters in critical velocity equation are expected to be constant for a given system, the variation in critical velocity can then be attributed either due to variation in $\Delta \sigma$ for individual particle and matrix or presence of convection. In the limited experiments conducted under $\mu \mathrm{g}$, where the effect of convection is mitigated the results show overall reduction in values of critical velocity but the trends from the data show possible presence of range of critical velocity.

The presence of ranges of critical velocity perhaps points to the fact that $\Delta \sigma$ does not have a unique value for all the particles in the matrix. This may be attributed to the presence of interfacial gaps between the particle and matrix, presence of impurities on the surface of particles or precipitation of reaction products on the particles. Any of these effects can cause variation in $\Delta \sigma$ for individual particles. However, the range of variation is quite small in the carefully conducted experiments. Therefore it is suggested that critical velocity equation can also be used to calculate interfacial energy if critical velocity is known. The main issue here is the accuracy of the model. The model in a modified form has been validated by conducting experiments in organic systems, viz. succino-nitrile-polystyrene and biphenyl-glass (Sen et al 1997).

\section{Summary and conclusions}

Interfacial energy between reinforcement and matrix in metal-matrix composites is a key issue that needs to be characterized to manufacture tailor-made engineering materials. A perfect interface is expected to give near ideal interface energy values. The direct measurement of interfacial energy poses serious experimental problems. Indirect characterization through correlation with critical velocity of engulfment by solidifying interface, calculated through validated models and measured through carefully conducted experiments seems to be a good option. However, interfacial energy and critical velocity calculation is a two-way process and each needs to be tuned to the other through active experimentation and fine tuning of critical velocity models. It is a subject that needs understanding physics and chemistry of the particle and the matrix interface in depth, careful experiments on ground and possibly in $\mu \mathrm{g}$. Interactive studies of this kind can only lead to the development of composites for high technology application.

\section{Acknowledgements}

The author sincerely acknowledges the contribution of Dr D M Stefanescu and Frank Juretzko, The University of Alabama, USA and Dr S Sen and Dr P Curreri, Space Science Laboratory, Huntsville, USA to the development of science and experimentation involved.

\section{References}

Adamson A W 1982 Physical chemistry of surfaces (New York: John Wiley \& Sons Inc.)

Bolling G F and Cisse J 1971 J. Cryst. Growth 1056

Chemov A A, Temkin D E and Melnikova A M 1976 Sov. Phys. Crystallogr. 21369

Endo A, Chauhan H S, Egi T and Shiohara Y 1996 J. Mater. Res. 11795

Jackson K A and Chalers B 1958 J. Appl. Phys. 291178

Juratzko F R, Dhindaw B K, Stefanescu D M, Sen S and Curreri P A 1998 Metall. \& Mater. Trans. A29 1691

Kacar A S, Rana F and Stefanescu D M 1991 Mater. Sci. \& Eng. A135 95

Kennedy A R and Clyne T W 1991 Cast Metals 4160

Kingrey W D 1954 J. Am. Ceram. Soc. 3742

Korber C, Rau G, Cosman M D and Cravalho E G $1985 \mathrm{~J}$. Cryst. Growth 72649

Lawrynowicz D E, Li B and Lavemia J 1997 Metall. Trans. B28 77

Li J G 1994 Ceram. Int. 20391

Murr L R 1975 Interfacial phenomena in metals and alloys (MA: Addison Wesley)

Nair S V, Tien J K and Bates R C 1985 lnt. Mater. Rev. 30275 Oh S Y, Cornie J A and Russel K C 1987 Metall. Trans. A20 527

Omenyi S N and Neumann A W 1976 J. Appl. Phys. 473956

Omenyi S N, Neumann A W and Van Oss C J $1981 \mathrm{~J}$. Appl. Phys. 52789

Potschke J and Rogge V 1989 J. Cryst. Growth 94726

Rohatgi P K, Ray S, Asthana R and Narendranath C 1993 Mater. Sci. \& Eng. A162 163

Sen S, Dhindaw B K, Stefanescu D M, Catalina A and Curreri P A 1997 J. Cryst. Growth 173574

Stefanescu D M, Dhindaw B K, Kacar S A and Moitra A 1988 Metall. Trans. A19 2847

Stefanescu D M, Moitra A, Kacar A S and Dhindaw B K 1990 Metall. Trans. A21 231

Stefanescu D M, Juretzko F R, Dhindaw B K, Catalina A, Sen S and Curreri P A 1998 Metall. \& Mater. Trans. A29 1697 Uhlman D R, Chalmers B and Jackson K A 1964 J. Appl. Phys. 352986

Wu Y, Liu H and Lavernia E J 1994 Acta Metall. Mater. 42 825 